\title{
Propuesta de expansor maxilar modificado para toda dentición
}

Valverde R, Camacho J. Propuesta de expansor maxilar modificado para toda dentición. Rev Estomatol Herediana 2006; 16(1): 53 - 58.

RESUMEN

El aparato de expansión rápida maxilar de Haas (o Hyrax) es muy versatil y ha sido objeto de distintas modificaciones de acuerdo a cada caso. En el presente artículo, presentamos dos propuestas que representan ventajas significativas. a) Utilizar los segundos molares deciduos superiores como piezas de anclaje principal del disyuntor, con las ventajas: inclinación y angulación más favorable que al utilizar los primeros molares permanentes, las piezas deciduas son perpendiculares al plano oclusal mientras que los primeros molares permanentes superiores erupcionan hacia vestibular y distal, Las raíces de los dientes deciduos son divergentes los que proveerían mayor anclaje, y los gérmenes de las piezas sucedáneas (segundos premolares) no se inclinarían, lo que si sucedería con los primeros molares permanentes. Se podría realizar con seguridad la expansión desde la dentición decidua o mixta primera fase. Recordemos que la terapia de ERM tiene mejores efectos a edad temprana. Los segundos molares deciduos son anatómicamente muy similares a los primeros molares permanentes, están en una posición más mesial y completamente erupcionados, lo que favorece la colocación de bandas sobre ellos. b) Soldar un tubo ortodóntico doble $\left(0,022 " \mathrm{x} 0,028 " \mathrm{x} 0,45^{\prime \prime}\right)$ por vestibular de la banda, con la intención de una vez finalizada la etapa activa de la expansión maxilar y estabilizado el tornillo expansor, cortar el arco de alambre y liberar las superficies vestibulares para instalar brackets (un arco utilitario o aparatología completa dependiendo de la dentición) y/o utilizar el tubo 0,45 para terapia con arco extraoral.

Palabras clave: TÉCNICA DE EXPANSIÓN PALATINA

\section{Correspondencia}

Renzo Valverde Montalva Carlos Tenaud 164 Of. 204 - Lima 33, Perú Teléfono: 4492494

e-mail: renzovm@yahoo.com

\section{Aceptado para publicación :}

16 de mayo del 2006.
Proposal of a modified maxillar expander for all the dentitions.

\section{ABSTRACT}

The Haas Rapid Maxillary Expansion (RME) appliance or Hyrax is a very versatile orthodontic device that could be modified for each case. This article presents two proposals with significant advantages. a) To use the upper deciduous second molar as the principal anchoring teeth of the disjunctor, considering that deciduous teeth are perpendicular to the occlusal plane, while the upper permanent first molars have a buccal and distal direction during the eruption. On the upper permanent first molars have a buccal and distal direction during the eruption. On the germs of the second premolars wouldn't suffer inclination in the same way that permanent first molars. Therefore, secure expansion is possible to perform using the deciduous dentition or during the first phase of the mixed dentition. RME therapy work better in early age. The deciduous second molars are anatomically very similar to permanent first molars, also is mesial position help the band placement. b) Soldering a double orthodontic tube $(0,022 " \mathrm{x} 0,028$ "x0,45") in the vestibular surface of the band when active stage of maxillary expansion is finished and the expansion screw is stabilized; the arch wire is cut and the buccal surfaces are free to install brackets (an utilitarian arch or complete aparatology depending on the dentition) and/or a 0,45 tube for extraoral arch therapy.

Keywords: PALATAL EXPANSION TECHNIQUE

\section{Expansión maxilar}

La expansión rápida de maxilar (ERM), es un procedimiento ortopédico relativamente común en ortodoncia que consiste en la apertura de la sutura medio palatina. La evidencia clínica es la apertura de un diastema entre los incisivos centrales superiores durante los primeros días (1).

Las indicaciones según Haas (2) para la ERM son:

1. Casos de Clase III por deficiencias maxilares, quirúrgicas y no quirúrgicas.

2. Casos de deficiencias maxilares reales y relativas (mordida cruzada uni o bilateral completa).

3. Casos de capacidad nasal disminuída con problemas respiratorios na- sales crónicos.

4. Casos de paladar fisurado completo.

5. Problemas seleccionados de forma de arco.

Las fuerzas utilizadas en la ERM, son fuerzas pesadas e intermitentes que en una simple activación puede llegar a 4,5kg y que múltiples activaciones diarias como se realiza usualmente, pueden acumular fuerzas residuales de hasta $9 \mathrm{~kg}$ (3), permitiendo que la fuerza aplicada en el proceso alveolar exceda los límites necesarios para el movimiento ortodóntico y promoviendo la separación de los dos hemimaxilares, cumpliendo por tanto, el rol de aparatos ortopédicos (2).

El momento ideal para la RME es en la dentición decidua o mixta primera fase. La sobrecorrección y la retención de los arcos dentales son recomendados para evitar una recidiva significativa (1). A mayor edad del paciente, mayor recidiva, por lo que la mayor recidiva se observa en pacientes puberales que en pacientes niños.

Los diferentes diseños traen ventajas inherentes y el clínico debe escoger el aparato, dependiendo de cada caso y los objetivos de tratamiento. Los aparatos de expansión adheridos, con las caras oclusales cubiertas por acrílico, demuestran ciertas ventajas sobre los expansores convencionales (bandeados), especialmente en casos donde no es deseable la inclinación de los dientes de soporte, sin embargo, las piezas 
donde se ha adherido el aparato deberán estar completamente erupcionadas para mejorar el anclaje (4). Un estudio demuestra que los aparatos de expansión maxilar rápida adherida, no solamente proporcionan control vertical, sino además separan las mitades maxilares en cuerpo entero (5).

Para la confección del Hyrax existen diseños con cuatro bandas (en molares o premolares) o solamente con dos, en primeras molares permanentes (6). El aparato Hyrax con cuatro bandas, es indicado cuando existe un severo apiñamiento anterior acompañado por un estrechamiento del arco, debido a que es deseable un aparato lo mas rígido posible y un diseño de Hyrax con dos bandas, es recomendado en dentición mixta, apiñamiento leve y constricción posterior (7).

Se han diseñado también aparatos de expansión añadiéndoles dispositivos como rejilla interceptora de hábitos (4).

Existen también expansores con diseños en alambre NiTi, para expansión lenta, en un estudio comparativo con el Hyrax, se concluye que en dentición decidua y mixta temprana ambos son capaces de expandir la dentición maxilar y el proceso alveolar, y por consiguiente, son capaces de corregir mordidas cruzadas posteriores, pero la expansión con Hyrax es mas confiable, el expansor de NiTi inclina más los molares labialmente y causa mayor rotación distal de la molar (8).

La única ventaja que ofrece el aparato Hyrax, es que al estar compuesto por una parte acrílica, se puede agregar dispositivos como springs, ganchos o rejillas. Es importante señalar que la principal desventaja, además del problema de higiene, del Hyrax es que el acrílico hace al aparato más flexible a las fuerzas pesadas necesarias para la disyunción.

\section{Expansión rápida del maxilar en denti- ción decidua y mixta}

da Silva en 1991 (9), propuso que las mordidas cruzadas posteriores deben ser corregidos lo mas temprano posible aun en dentición decidua. Propone el uso de un aparato de Haas con una modificación, un retenedor de tipo circunferencial en canino deciduo que se sostiene con resina en lugar de bandas.

Este dispositivo esta indicado en dentición decidua y mixta cada vez que la mordida cruzada esta asociada con la constricción esquelética, la cual puede ser identificable como:

1. Mordida cruzada posterior uni o bilateral con inclinación normal del proceso alveolar.

2. Mordida cruzada posterior uni o bilateral con retrusión del tercio medio facial.

3. Mordida cruzada completa.

Las ventajas de tomar el segundo molar deciduo como pilar para la banda son :

- La disposición divergente de las raíces, dan más estabilidad en el momento de la disyunción.

- El segundo molar deciduo se encuentra más perpendicular al plano oclusal. En la primera etapa de dentición mixta el primer molar permanente se encuentra notablemente vestibulari-zado y es por tanto menos elegible para la aplicación de fuerzas, ya que esto contribuiría a una mayor inclinación bucal de la corona, mientras que si nos apoyamos en el segundo molar deciduo, el sucedáneo (segundo premolar) no se verá afectado.

- El primer molar permanente es menos accesible y está menos erupcionado para la adaptación de la banda que el segundo molar deciduo.

\section{Descripción del expansor propuesto}

Presentamos un diseño versátil de aparato de disyunción, que puede ser usado en dentición decidua, mixta y permanente. Consta de dos bandas adheri- das en segundos molares deciduos o primeras molares permanentes (en caso de dentición permanente). La banda lleva soldado un tubo doble $(0,022 " \mathrm{x}$ $0,028 "$ x 0,45"/ 0,51"). Un alambre circunferencial (1,2 mm) que se extiende hasta la pieza mas mesial (caninos temporarios, primeros molares deciduos, caninos permanentes o premolares permanentes, según el caso), el alambre circunscribe estas piezas por el ecuador y en dirección vestibular llegando finalmente al tubo soldado en la banda, entrando por el tubo 0,45"/0.51", doblándolo en distal.

El tornillo tipo Hyrax es higiénico, de gran rigidez, y capaz de soportar fuerzas pesadas. Tanto el tornillo de Hyrax como de Haas están disponibles en presentaciones 9, 11, y 13 mm. La ubicación del tornillo en el paladar, será siempre en la línea media y funciona mediante una llave de activación la cual permite abrir el tornillo a razón de $0,25 \mathrm{~mm}$ por activación, el protocolo de activación es como sigue : dos activaciones en la mañana y dos activaciones en la noche (es decir un milímetro por día), el tornillo se activará tres días consecutivos, y se deja de activar dos días intercaladamente para disipar las fuerzas residuales (3), se repite el esquema de activación hasta que la cúspide palatina superior contacte con la cúspide vestibular inferior.

Una vez instalado, el aparato resulta bastante rígido, estable, y versátil permitiéndonos poder colocar aparatos $4 \times 2$ inmediatamente después de realizada la expansión maxilar o colocar aparatos completos tratándose de denticiones permanentes (luego de terminada la disyunción, el alambre circunferencial se puede seccionar, permitiendo colocar brackets en los premolares y caninos sin necesidad de retirar el aparato de expansión), ya que los alambres ortodónticos podrán ser anclados en el tubo 0,022 " x 0,028". Otra de las ventajas es que al tener sólo dos bandas, se minimiza el tiempo de trabajo. 


\section{Casos clínicos}

\section{Caso I}
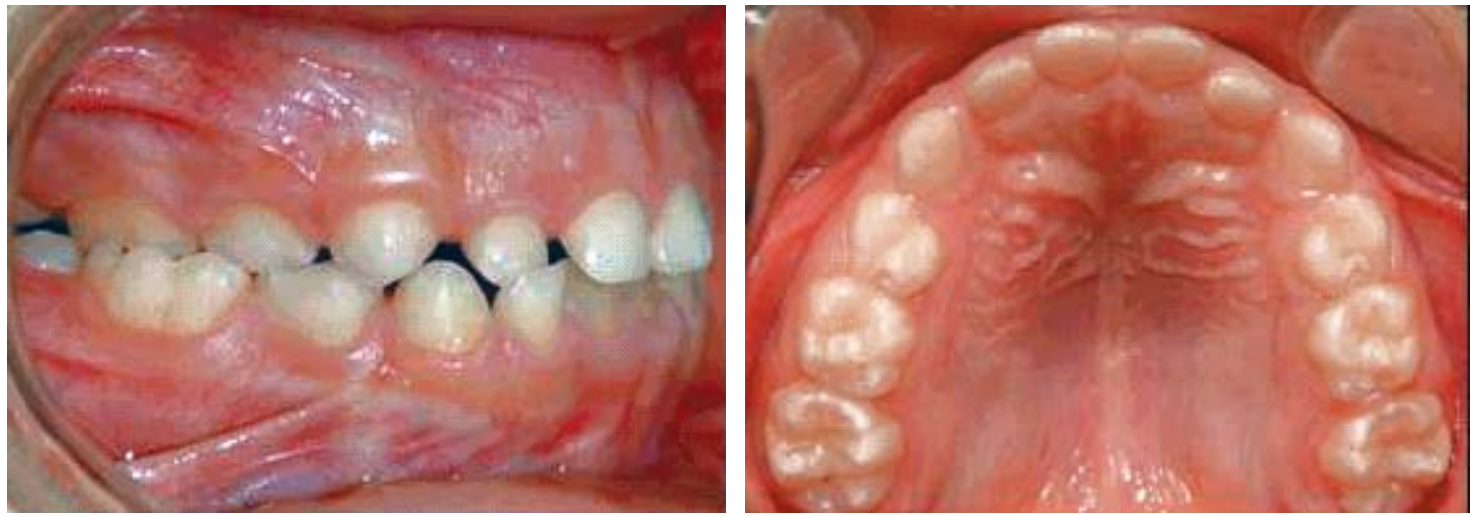

Fig 1. Clase I en dentición mixta primera fase incompleta por mordida cruzada completa
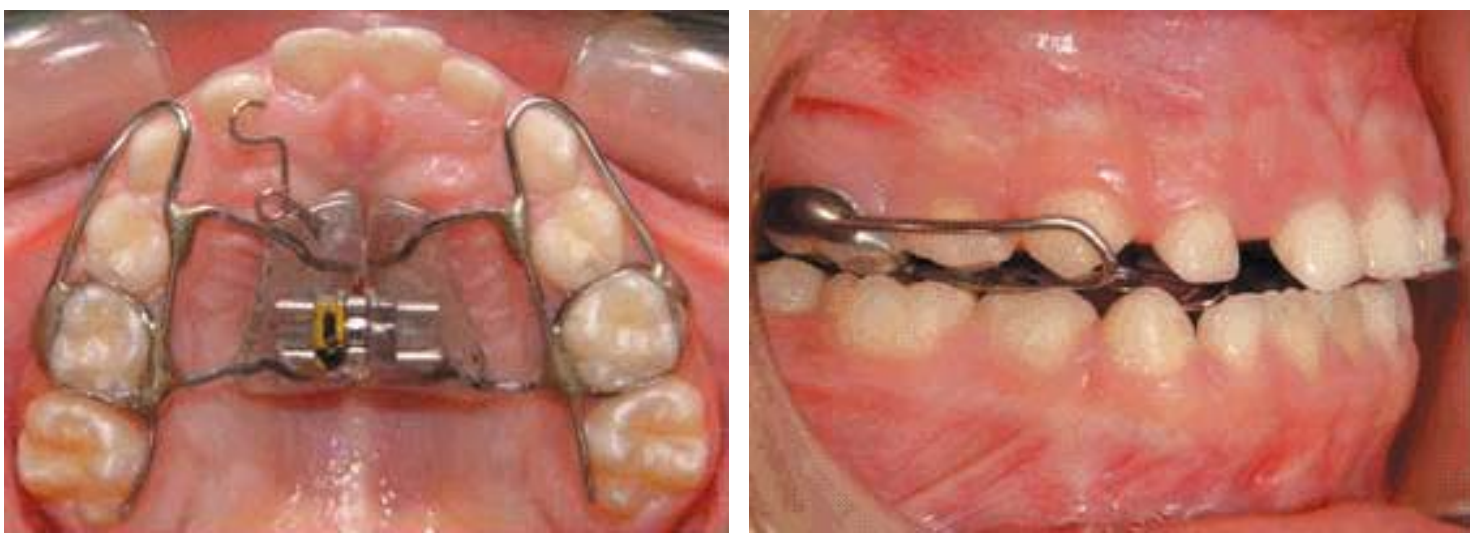

Fig 2. A. ERM Haas modificado, antes de activación, nótese la construcción de bandas sobre los segundos molares deciduos y las extensiones para los primeros molares permanentes. B. ERM Haas después del periodo de activación, nótese la sobreactivación (hasta que contacten las cúspides palatinas con las vestibulares)
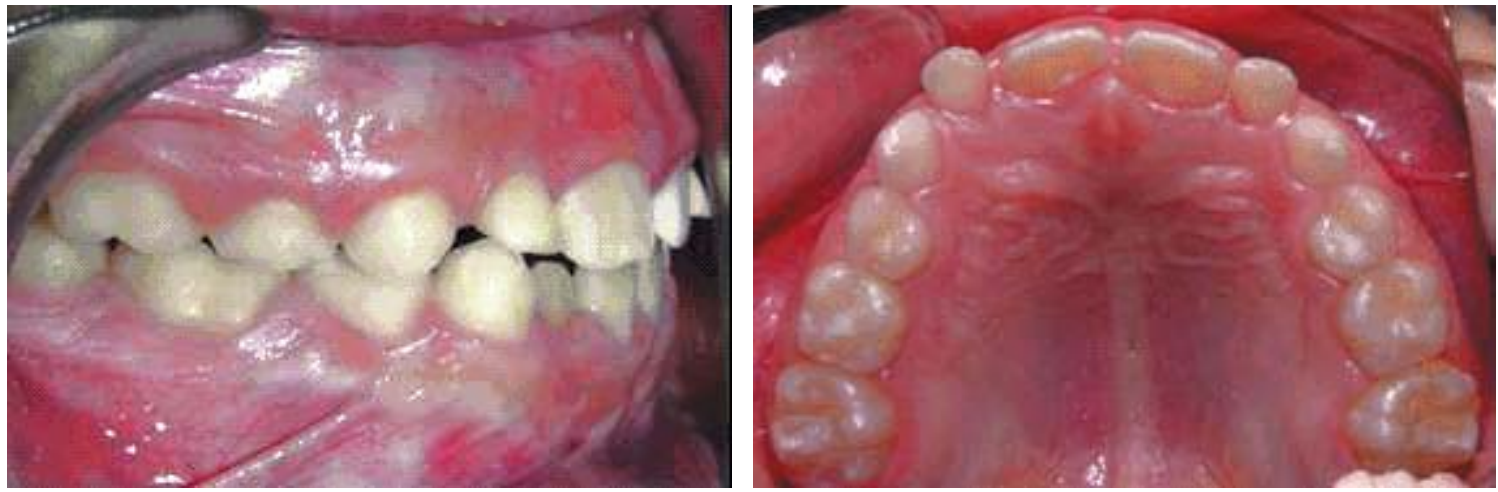

Fig 3. Estabilidad del caso seis meses después de retirado el aparato 


\section{Caso II}
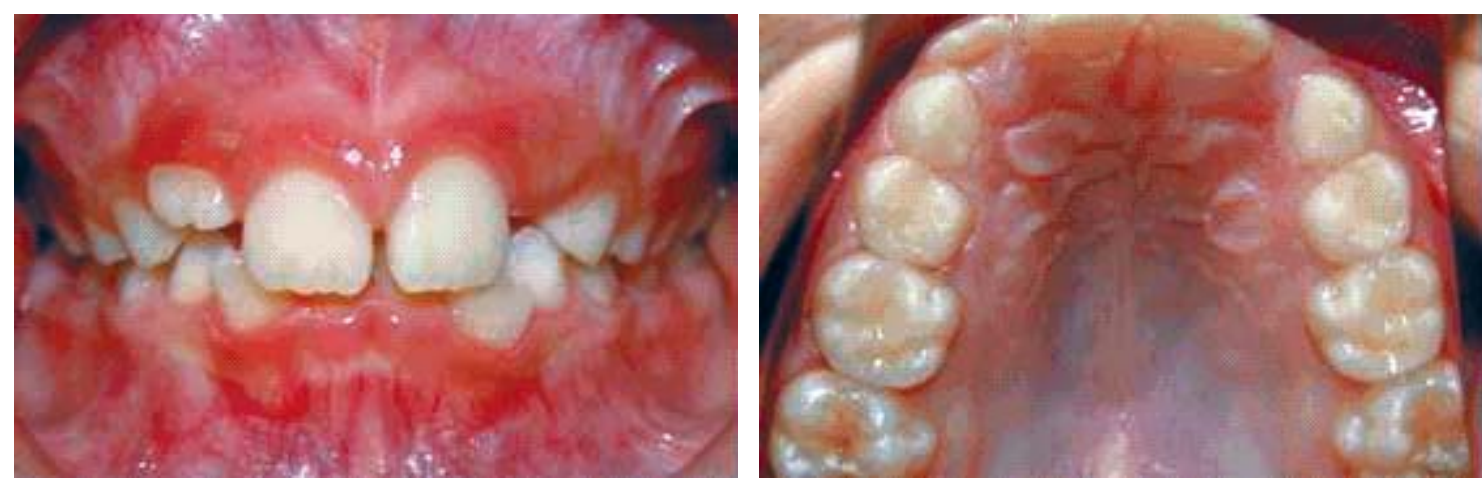

Fig. 4. Clase I en dentición mixta primera fase incompleta por falta de espacio para el descenso de los incisivos laterales permanentes
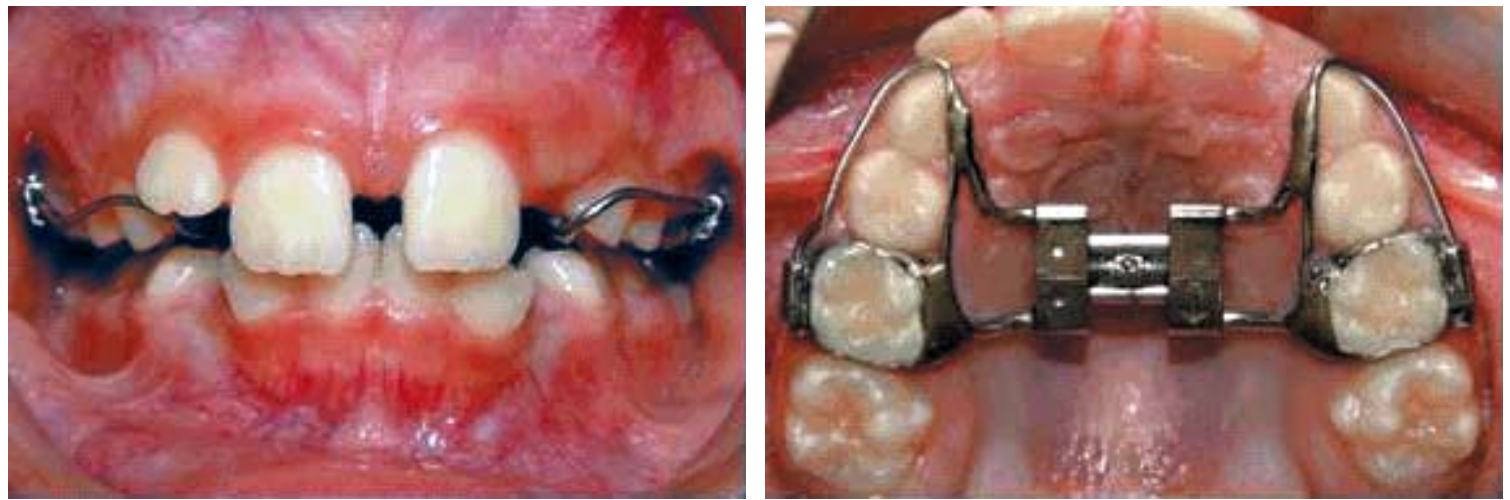

Fig. 5. ERM Hyrax después del periodo de activación, nótese el diastema signo de la disyunción, la sobreactivación del aparato, la construcción de bandas sobre los segundos molares deciduos, y los tubos dobles.
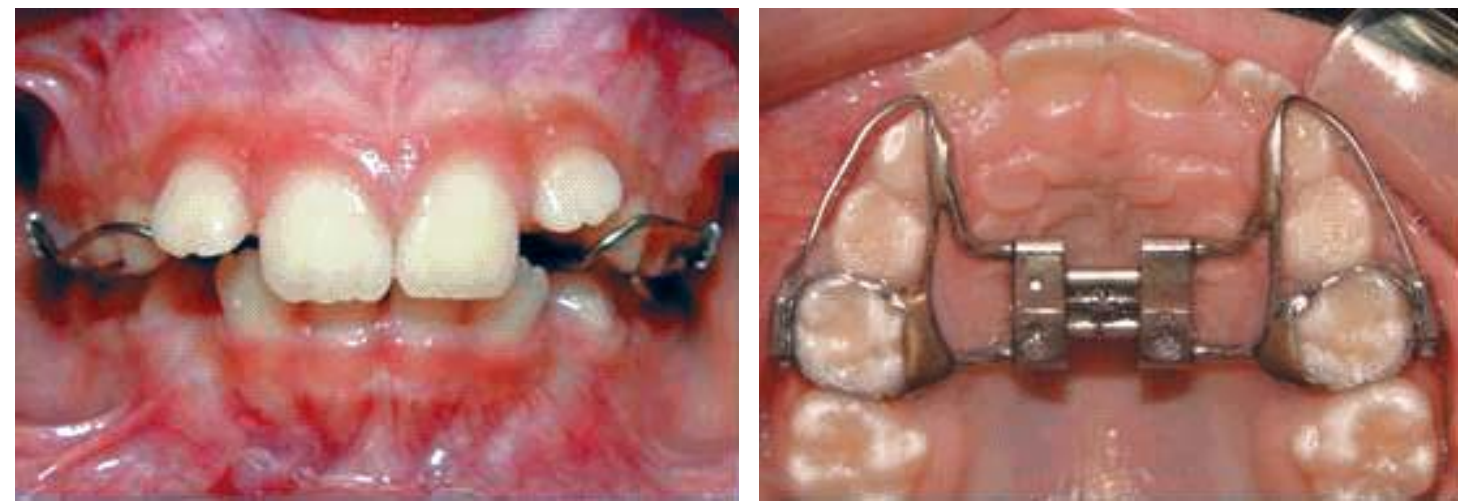

Fig. 6. A. Descenso espontáneo de los Incisivos Laterales Permanentes posterior al aumento de perímetro. Nótese el cierre espontáneo del diastema. B. ERM Hyrax después del periodo de activación, nótese el cierre del diastema signo de la disyunción, y la sobreactivación del aparato.

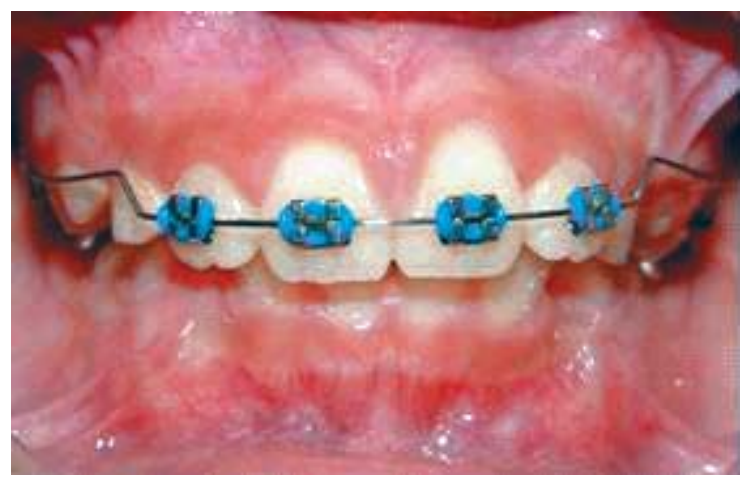

Fig. 7. Después del periodo de activación se instaló un aparato 4x2 para finalizar el alineamiento y nivelación 


\section{Caso III}
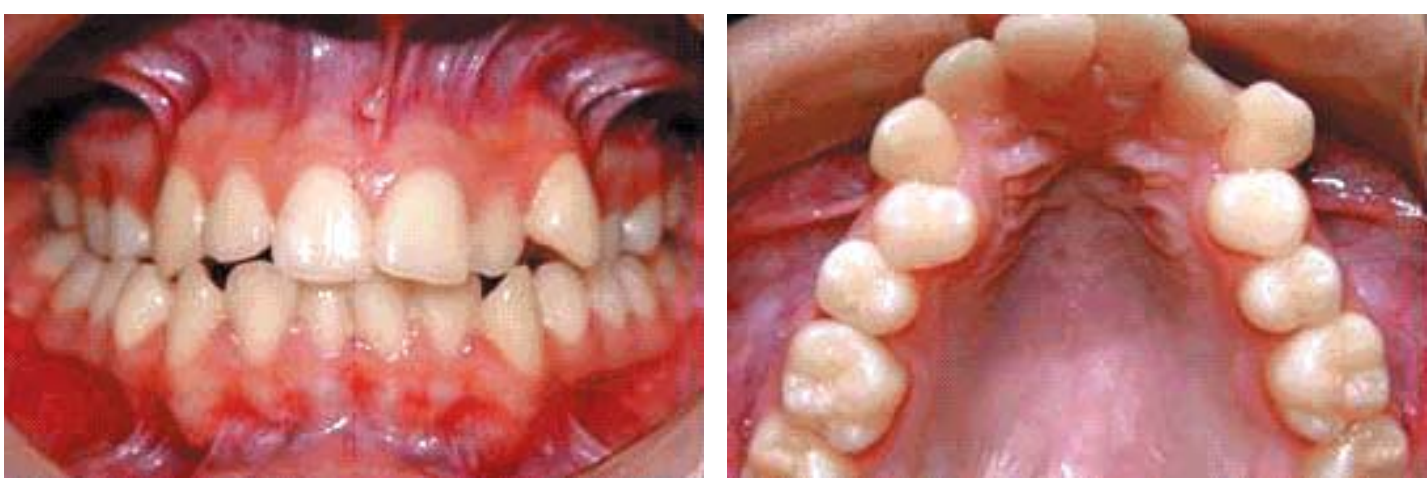

Fig. 8. Clase I por falta de espacio y mordida cruzada bilateral en dentición permanente. Nótese la constricción del arco maxilar
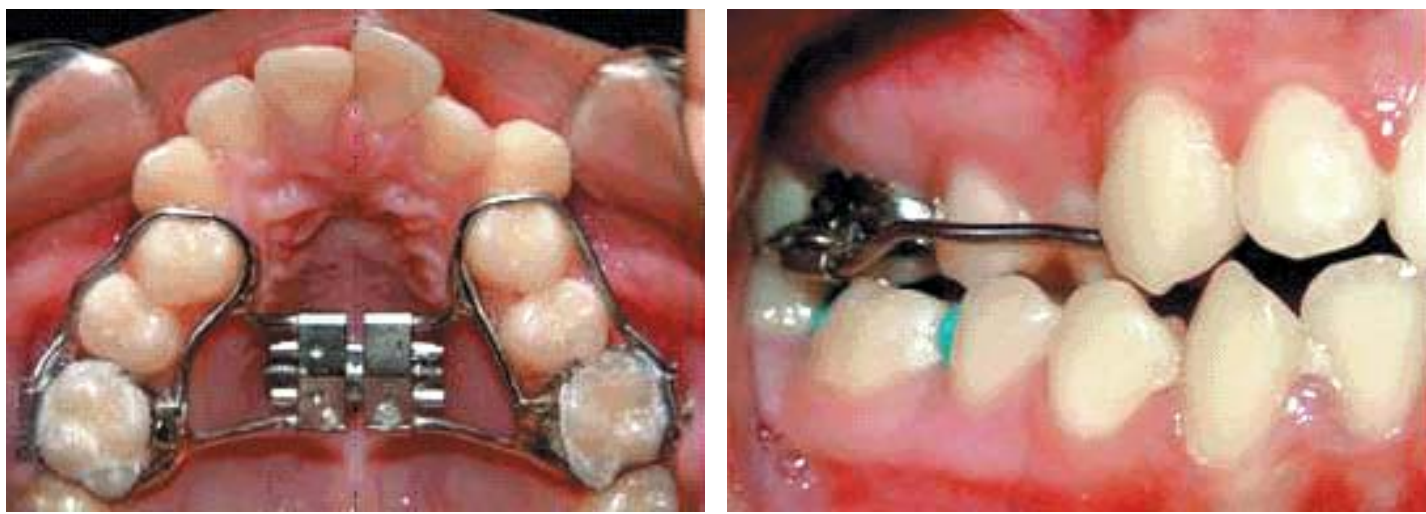

Fig. 9. ERM Hyrax modificado antes del periodo de activación, nótese los tubos dobles en las bandas de los molares, a la derecha detalle del tubo, el alambre $0,12 \mathrm{~mm}$ se dobla a distal del tubo
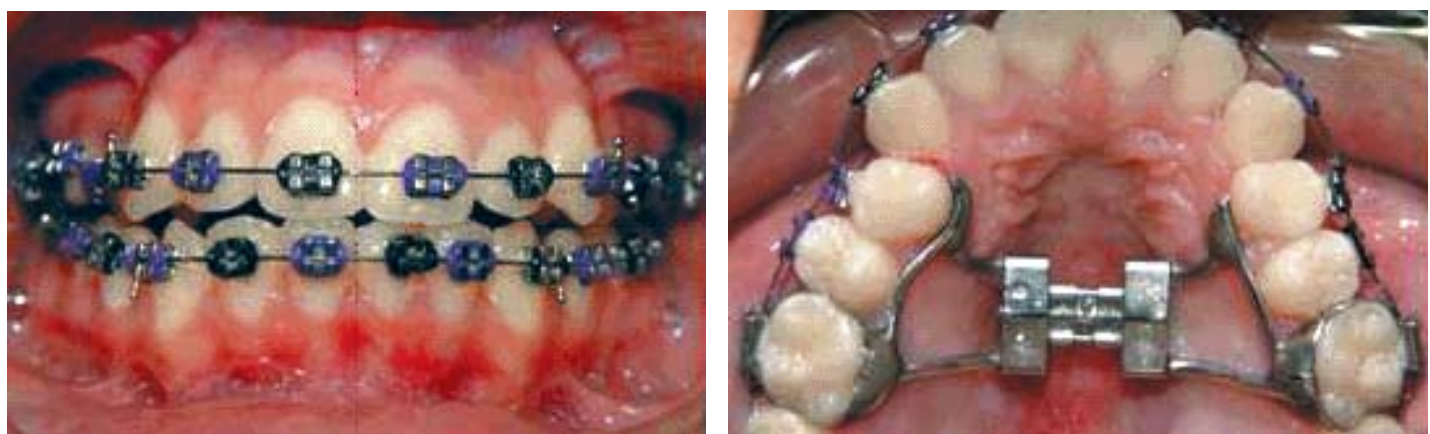

Fig. 10. Después del periodo de activación (21 días), se secciona la parte vestibular del aparato a la altura del primer premolar para permitir la colocación de brackets durante el periodo de estabilización de la expansión (5 meses), además el Hyrax servirá como contención
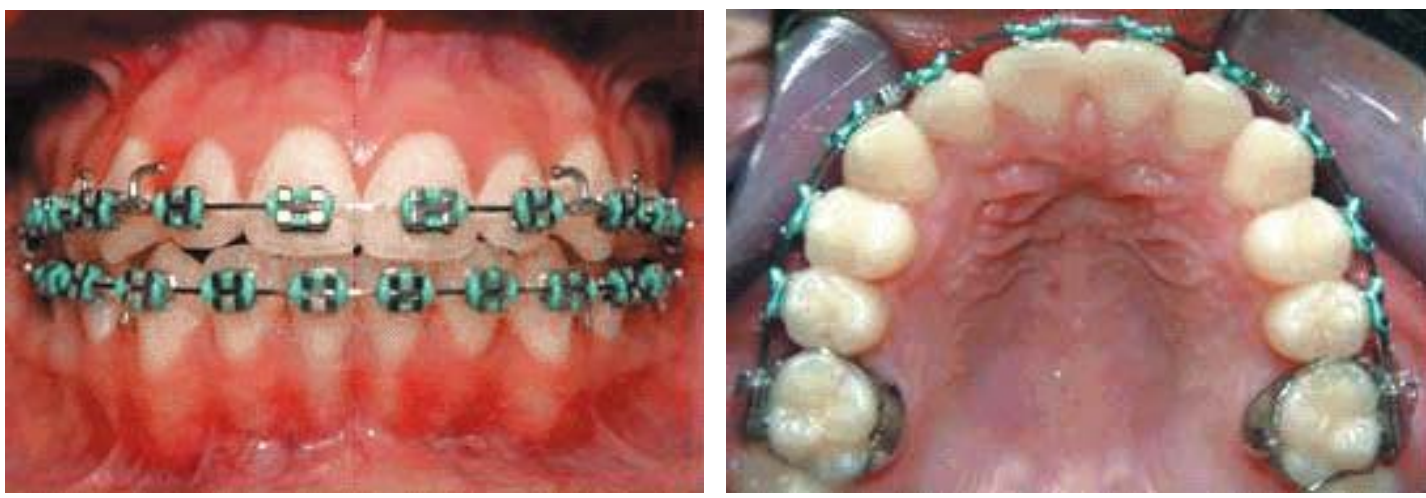

Fig. 11. Final del periodo de estabilización. 


\section{Referencias bibliográficas}

1. Haas A. The treatment of maxillary deficiency by opening the midpalatal suture. Angle Orthod 1965; 35:200-17.

2. Haas A. Rapid expansión of the maxillary dental arch and nasal cavity by opening of the midpalatal suture. Angle Orthod 1961; 31:73.

3. Isaacson R, Wood J, Ingram L. Forces pruduced by rapid maxillary expansion: Design of a force measuring system. Angle Orthod 1964; 34:256-60.

4. Memikoglu TU, Iseri $\mathrm{H}$. Effects of a bonded rapid maxillary expansion appliance during orthodontic treatment. Angle Orthod 1999; 69(3):251-6.

5. Spolyar JL. The design, fabrication, and use of a full-coverage bonded rapid maxillary expansion appliance. Am J Orthod 1984; 86(2):136-45.

6. Davidovitch M, Efstathiou S, Sarne O, Vardimon AD. Skeletal and dental response to rapid maxillary expansion with 2 - versus 4-band appliances. Am J Orthod Dentofacial Orthop 2005; 127(4):483-92.

7. Viazis AD, Vadiakis G, Zelos L, Gallagher RW. Designs and appli- cations of palatal expansion appliances. J Clin Orthod 1992; 26(4):239-43.

8. Ciambotti C, Ngan P, Durkee M, Kohli $\mathrm{K}, \mathrm{Kim} \mathrm{H}$. A comparison of dental and dentoalveolar changes between rapid palatal expansion and nickel-titanium palatal expansion appliances. Am J Orthod Dentofacial Orthop 2001; 119(1):11-20.

9. da Silva Filho OG, Boas MC, Capelozza Filho L. Rapid maxillary expansion in the primary and mixed dentitions: a cephalometric evaluation. Am J Orthod Dentofacial Orthop 1991; 100(2):171-9. 